\title{
Madurez neuropsicológica y uso de las TIC en el aprendizaje del inglés
}

\section{Miriam Romero López ${ }^{1}$, Montserrat López Fernández ${ }^{1}$ y María Carmen Pichardo Martínez ${ }^{1}$}

\footnotetext{
${ }^{1}$ Departamento de Psicología Evolutiva y de la Educación, Universidad de Granada
}

\section{España}

Correspondencia: Mirian Romero López. Facultad de Ciencias de la Educación. Campus Universitario de Cartuja s/n, 18071. Granada (España). E-mail: miriam@ugr.es

(C) Universidad de Almería and Ilustre Colegio Oficial de la Psicología de Andalucía Oriental (Spain)) 


\section{Resumen}

Introducción: El gran impacto tecnológico de las últimas décadas y el amplio desarrollo de las Tecnologías de la Información y Comunicación (TIC) se han establecido como una característica propia de la sociedad actual. Este avance vertiginoso, y la cantidad y diversidad de cambios que se van generando en el conocimiento, están induciendo a la escuela y a su currículum a una importante innovación y transformación. La irrupción de las TIC en el ámbito educativo conlleva un incremento en la motivación y el interés de los procesos de aprendizaje en el alumnado. Asimismo, han fomentado el desarrollo de una mayor autonomía y competencia dentro de esta sociedad en continua transformación. Por otro lado, la cantidad de tiempo que el alumnado dedican al uso de determinadas tecnologías (teléfono móvil, internet, redes sociales y videojuegos) fuera del contexto escolar y la variedad de recursos digitales y audiovisuales que se están desarrollando, lleva a plantearse su influencia en algunos de los procesos psicológicos básicos para el aprendizaje, y su pertinencia para la práctica docente. Se ha demostrado que la utilización de las TIC influye en habilidades como la atención, la percepción o la memoria. Por esta razón, la presente investigación pretende: a) analizar la influencia de las TIC en el aprendizaje de segundas lenguas, concretamente en el inglés, b) comprobar si existe relación entre la madurez neuropsicológica y el aprendizaje de segundas lenguas, c) conocer el papel que juega la atención en el aprendizaje de segundas lenguas y d) indagar si el aprendizaje de las segundas lenguas a través de las TIC influye en la motivación del alumnado.

Metodo: En el presente estudio participaron 70 alumnos y alumnas, 35 niñas y 35 niños, de edades comprendidas entre 10 y 11 años, matriculados en $5^{\circ}$ curso de Educación Primaria de un centro concertado de la ciudad de Granada.

Resultados: Los resultados muestran que tanto la metodología empleada a través de TIC como el desarrollo neuropsicológico del alumnado influyen en el aprendizaje del inglés como segunda lengua. Igualmente, la atención también se relacionó con el aprendizaje del inglés. Por otro lado, la motivación hacia el aprendizaje fue mayor en el alumnado que aprendió a través de las TIC.

Discusión y conclusiones: Por lo tanto, se podría concluir que una metodología basada en las TIC favorece el aprendizaje de las segundas lenguas, así como la motivación del alumnado hacia el aprendizaje. Igualmente, los estudiantes con mayor desarrollo neupsicológico y con puntuaciones más elevadas en atención tienen más facilidad para el aprendizaje del inglés.

Palabras Clave: Tecnologías de la Información y de la Comunicación (TIC), aprendizaje del segundo idioma, inglés, motivación, atención, desarrollo neuropsicológico, funciones ejecutivas. 


\begin{abstract}
Introduction: The great technological impact of recent decades and the widespread development of Information Technology and Communication (ICT) have been established as a feature of modern society. This vertiginous advance and the amount and variety of changes that have been generated in our knowledge, are leading school and its curriculum to an important innovation and transformation. The emergence of ICT in education, has led to a greater increase in motivation and interest of students in learning processes. Similarly, they have encouraged the development of greater autonomy and competence within this society that it is showed in continuous transformation. Furthermore, the amount of time that students spend using particular technologies (mobile, internet, social networking and video games) outside the school context and the variety of audiovisual and digital resources that are being developed, raise the question of its influence on some of the basic psychological processes for learning and its relevance to teaching practice. It has been shown that the use of ICT affects skills such as attention, perception or memory. For this reason, the present research aims to: a) analyze the influence of ICT in the learning of second languages, specifically in English, b) check if there is a relationship between neuropsychological maturity and second language learning, c) know the role that the attention plays in the learning of second languages and d) to investigate if the learning of the second languages through the TIC influences in the motivation of the alumnado.
\end{abstract}

Method: In the present study 70 students were included, 35 girls and 35 boys, aged between 10 and 11 years, enrolled in 5th year of primary education in a private publicly funded school in Granada.

Results: The results show that both the methodology employed by ICT and the neuropsychological development influence learning English as a second language. Likewise, the attention was also related to learning English. On the other hand, the motivation towards learning was greater in the students who learned through ICT.

Discussion or Conclusion: Therefore, it could be concluded that a methodology based on ICT favors the learning of second languages, as well as the motivation of students towards learning. Likewise, students with greater neupsychological development and higher scores in attention have more facility for learning English.

Keywords: Information and Communication Technology (ICT), second language learning, English, motivation, attention, neuropsychological development, executive function. 


\section{Introducción}

Actualmente, el impacto de las nuevas tecnologías en la sociedad ha generado cambios en la mayor parte de la actividad humana (Hernández y Muñoz, 2012). La emergente sociedad de la información y el desarrollo tecnológico también han dado lugar a cambios en el proceso educativo (Hubackova y Ruzickova, 2011). En los últimos años se ha producido una mayor preocupación por la utilización eficaz de los diversos recursos informáticos y de internet en los distintos niveles educativos, así como en los diversos contextos formales e informales. Por ello, multitud de profesorado, investigadores e investigadoras han estado interesados e interesadas en la influencia de las nuevas tecnologías en el aprendizaje y, más concretamente, en el uso de las tecnologías de la información y la comunicación (TIC) en la enseñanza de lenguas extranjeras (Ghasemi y Hashemi, 2011; Røkenes y Krumsvik, 2016).

Según Ghasemi y Hashemi (2011) las TIC son definidas como un conjunto diverso de herramientas tecnológicas y los recursos utilizados para comunicarse, y para crear, difundir, almacenar y gestionar la información. La integración de tales tecnologías en el ámbito educativo ha generado variaciones en los procesos de enseñanza y aprendizaje (Yunus, Nordin, Salehi, Embi, y Salehi, 2014).

En la década de los 60, apareció el primer sistema informático y desde entonces se han implementado diversas medidas para incorporar las TIC en los distintos niveles educativos (Badia, Meneses, y Sigalés, 2013; Nordin, Embi, y Yunus, 2010; Nordin, Hamzah, Yunus, y Embi, 2010; Salehi y Salehi, 2012; Yunus, Nordin, Salehi, Embi, y Salehi, 2013; Yunus, Salehi, y Chenzi, 2012). Del mismo modo, a pesar de que la introducción de las TIC se produjo hace muchas décadas, aún sigue existiendo mucha controversia sobre su impacto en el aprendizaje del alumnado. La utilización de las TIC como herramienta educativa presenta algunas limitaciones tales como el elevado coste, la escasa disponibilidad de equipos, la falta de conocimiento sobre su uso y la reticencia de algunos docentes para aceptar las TIC como recurso educativo (Harutyunyan, Moldoveanu, Moldoveanu, y Asavei, 2015).

Sin embargo, el motivo por el cual las TIC están adquiriendo tanta importancia en el ámbito educativo radica en que diversas investigaciones muestran que aumentan la calidad de la educación, permiten el acceso a una gran cantidad de información de forma rápida e incrementan el interés y motivación hacia el aprendizaje, entre otras (Ghasemi y Hashemi, 2011). 
Así pues, existe una necesidad de realizar investigaciones sistemáticas acerca del impacto de las TIC en la educación, puesto que a pesar de que éstas se han integrado con éxito en el aula, poco se sabe sobre su impacto directo sobre en el logro académico (Condie y Munro, 2007). La mayoría de los estudios se han centrado en analizar la relación entre las TIC y la mejora de la motivación (Passey, Rogers, Machell, y McHugh, 2004; Yunus, Chua, Maimun, y Rizauddin, 2010), una variable que se considera básica para el logro académico y la mejora del aprendizaje.

Por otro lado, en los últimos años, el dominio de otras lenguas está siendo de un objetivo prioritario dentro del contexto académico (Matsuda y Friedrich, 2011; McKay, 2002). Según Abdel-Rahman (2009) el chino y el inglés son los idiomas de mayor uso, utilizándose el inglés como idioma internacional gracias a la globalización y la competencia entre las naciones (Faradilla, 2010). El inglés como idioma internacional hace referencia a un termino que describe la función o el uso del inglés en contextos multilingües (Friedrich y Matsuda, 2010; Matsuda y Friedrich, 2011). Es aquí donde empieza a surgir la necesidad de introducir el inglés dentro del plan de estudios de las escuelas (Shamsudin, 2009), puesto que, al ser una lengua internacional, se destaca la importancia de su enseñanza (Emir, 2010).

No obstante, en la actualidad, la mayor parte de la población no domina el uso del inglés a pesar de estar incluido en el plan de estudios desde el primer curso de primaria (Sekharan et al., 2014). Esto puede deberse a que la instrucción y la evaluación a la que se somete el alumnado es pasiva y no tiene en consideración las diferencias individuales y las necesidades de aprendizaje de cada alumno y alumna en particular (Arteaga, 2011; Prato y Mendoza, 2006). Todo esto genera sentimientos de frustración y un gran reto para el profesorado, puesto que varias publicaciones y conferencias recogen que la forma de enseñar inglés del profesorado es inadecuada pero, por otro lado, no brindan soluciones a esta problemática (Matsuda y Friedrich, 2011).

Hace algunos años, la enseñanza de segundas lenguas se basaba en la enseñanza tradicional. Sin embargo, hoy en día, la enseñanza debe tener en cuenta nuevos métodos como puede ser el uso de las TIC en el aula (Hubackova y Ruzickova, 2011). 
Así pues, debido a las múltiples ventajas que presentan las TIC, estas pueden ser una herramienta pedagógica trascendental en el ámbito educativo. Diversos estudios han demostrado que el uso de las TIC en el aula optimiza el aprendizaje de idiomas y aumenta la motivación y el interés de los estudiantes (Yunus et al., 2010). En esta línea, Passey et al. (2004) realizaron un estudio y encontraron que la utilización de las TIC en el aula genera altos niveles de motivación de logro en el alumnado.

Además, el uso de las TIC para el aprendizaje del inglés ayuda a potenciar las habilidades de lectura, escritura, habla y escucha. En un estudio realizado por Harrison et al. (2002), se encontró que las TIC contribuyen a un mayor compromiso del alumnado, a la reflexión, el análisis de la temática estudiada y favorece el desarrollo de competencias asociadas a la comunicación.

Muslem y Abbas (2017) mencionan una serie de ventajas de usar las TIC en el proceso de enseñanza y aprendizaje, especialmente en las clases de inglés. La primera, como se ha comentado anteriormente, es la motivación. Granito y Chernobilsky (2012) recogen que la tecnología tiene el potencial de ser una herramienta educativa poderosa y es probable que los estudiantes que usan la tecnología permanezcan realizando una tarea por períodos de tiempo más largos. Por ejemplo, en una clase de escucha, el profesorado puede usar internet para encontrar fuentes ricas de modelos orales nativos, que ayudan al alumnado con la pronunciación y también sirven de apoyo para el profesorado que no se sienta muy seguro con sus propias habilidades lingüísticas. En consecuencia, Silviyanti (2014) descubrió que el uso de YouTube parecía ser interesante y beneficioso para los estudiantes, ya que se entusiasmaban más y estaban más motivados por ver un video y luego practicar la pronunciación de las palabras como los hablantes nativos en lugar de solo practicar la escucha utilizando instrumentos de audio tradicionales.

La segunda es el aprendizaje colaborativo. Domalewska (2014) afirma que el aprendizaje colaborativo, apoyado por tecnología, puede impulsar el desarrollo del lenguaje porque los estudiantes aprenden dentro de las interacciones sociales. Por ejemplo, Silviyanti y Yusuf (2015) han encontrado que los blogs alientan a los estudiantes a hacer comentarios sobre el trabajo de los demás, a compartir sus experiencias, a reflexionar sobre el trabajo propio y sobre el de sus compañeros y compañeras, y así desarrollar sus habilidades de pensamiento crítico. 
La tercera es la comprensión cultural. Hollenbeck y Hollenbeck (2009) afirman que las TIC proporcionan al profesorado herramientas para abordar la equidad y los problemas de acceso, para acelerar el desarrollo lingüístico y conceptual de los estudiantes, para brindar apoyo a los estudiantes que aprenden de diferentes maneras y para crear experiencias de aprendizaje auténticas y significativas. La tecnología puede adaptarse a la comprensión cultural, en la que los estudiantes pueden interactuar y participar plenamente en su aprendizaje a medida que adquieren habilidades lingüísticas y conciencia cultural.

Por otro lado, el uso de las TIC en las aulas de inglés también tiene algunas desventajas. Kolbakova (2014) sugiere que el uso de las TIC en el proceso de enseñanza y aprendizaje agrega más trabajo y lucha adicional por parte del profesorado para satisfacer las necesidades de cada alumno y alumna en clase. Las TIC pueden no ser adecuadas para todos los estudiantes en todas las situaciones y propósitos, y, por lo tanto, requieren una considerable capacitación del alumnado para utilizarlas con éxito. Otras barreras para las TIC incluyen el control de clase y la distracción. Uno de los problemas con los que a menudo se enfrenta el profesorado que usa la tecnología se produce cuando los estudiantes copian y pegan de la fuente de Internet, también conocida como actividades de plagio. La facilidad para obtener información rápida y masiva de Internet hace que los estudiantes sean apáticos en la preparación de sus tareas. Yunus et al. (2013) explican con más detalle que las habilidades de lectura de los estudiantes pueden llevar a una comprensión inexacta del contenido cuando se desarrollan simplemente desplazando la pantalla del ordenador.

No obstante, las TIC en el aula para enseñar y aprender un idioma extranjero hoy en día son inevitables, ya que ahora son parte de nuestra vida diaria. Rabah (2015) ha afirmado firmemente el uso de la tecnología en el aprendizaje, ya que es una herramienta de aprendizaje poderosa y flexible que se necesita para enfrentar los desafíos de la globalización, promover el estado económico de un país y motivar y ayudar a los estudiantes a aprender.

Otra variable de especial relevancia en el aprendizaje es la madurez neuropsicológica, definida como el desarrollo madurativo y nivel de organización que permite el desenvolvimiento de las funciones conductuales y cognitivas de acuerdo con la edad cronológica de las personas. Cuando se aborda el concepto de madurez neuropsicológica, se incluyen procesos de desarrollo armónicos y progresivos en diferentes dimensiones como la lateralidad, visoper- 
cepción, función ejecutiva, ritmo y las habilidades perceptuales: atención, memoria y el lenguaje (Portellano, Mateos, Martínez, y Granados, 2000).

Las alteraciones neuropsicológicas frecuentemente están relacionadas con el fracaso escolar y con dificultades en el aprendizaje. Por esta razón, conocer el desarrollo neuropsicológico del alumnado permite establecer mejor sus posibilidades educativas con el fin de potenciarlas dentro del aula y prevenir el fracaso escolar (Diamond, 2013; Portellano, Mateos, y Martínez, 2012; Santa-Cruz y Rosas, 2017).

En esta línea, Martínez (2015) defiende que existe una relación entre el desarrollo neuropsicológico y los aprendizajes y por extensión, el aprendizaje de segundas lenguas. Sin embargo, en la actualidad se desconoce la existencia de estudios donde se evidencie el impacto de la madurez neuropsicológica sobre el aprendizaje de segundas lenguas, de ahí el interés de incluir esta variable en el presente estudio.

Por otro lado, la atención definida como el mecanismo implicado de forma directa en la activación y el funcionamiento de los procesos u operaciones de selección, distribución y mantenimiento de la actividad psicológica (García-Sevilla, 1997), también desempeña un papel muy importante en el aprendizaje de cualquier materia, puesto que es considerada como un componente vital para la selección y manipulación de la información (Laberge, 1995; León, 2008). Tradicionalmente, la atención ha sido considerada como un buen predictor del éxito académico. De esta manera, aquellos estudiantes que presentan una buena atención suelen obtener, a su vez, mejores resultados académicos. Sin embargo, el alumnado más inquieto, con una mayor tendencia a distraerse suele presentar puntuaciones más bajas en las pruebas de atención y un peor rendimiento académico (Boujon y Quaireau, 1999). Así mismo, los estudiantes con déficits atencionales presentan dificultades en la adquisición de la lectura y en la escritura (González-Castro, Álvarez, Núñez, González-Pienda, y Álvarez, 2008; León, 2008), habilidades que son prerrequisitos para cualquier aprendizaje curricular.

Por esta razón, el papel de la atención en la adquisición de un segundo idioma se ha convertido en un tema de gran interés en el ámbito de la investigación (Nelson, 2011; Robinson, 2003). En esta línea, Nelson (2011) demostró que la atención puede tener un papel fundamental en el aprendizaje de segundos idiomas. 


\section{Objetivos e hipótesis}

Teniendo en cuenta la importancia que el uso de las TIC, la atención, la madurez neuropsicológica y la motivación tienen en el aprendizaje, la presente investigación pretende: a) analizar la influencia de las TIC en el aprendizaje de segundas lenguas, concretamente en el inglés, b) comprobar si existe relación entre la madurez neuropsicológica y el aprendizaje de segundas lenguas, c) conocer el papel que juega la atención en el aprendizaje de segundas lenguas y d) indagar si el aprendizaje de las segundas lenguas a través de las TIC influye en la mo-tivación del alumnado.

Teniendo en cuenta los objetivos de investigación y la revisión teórica realizada se espera que a) el alumnado que recibe una enseñanza a través de TIC obtendrá mejores resultados en el aprendizaje de $2^{a}$ lenguas que sus iguales que reciben una enseñanza tradicional, b) el aprendizaje de $2^{\mathrm{a}}$ lenguas será superior cuanto mayor es la madurez neuropsicológica del alumnado, c) una puntuación alta en la atención se relacionará con un mayor aprendizaje de $2^{\mathrm{a}}$ lenguas y d) el alumnado que aprende a través de TIC tendrá una mayor motivación hacia las $2^{\mathrm{a}}$ lenguas que el alumnado que recibe una enseñanza tradicional.

\section{Método}

\section{Participantes}

En el estudio participaron 70 estudiantes, 35 niñas y 35 niños, de edades comprendidas entre 10 y 11 años, matriculados en el $5^{\circ}$ curso de Educación Primaria de un centro concertado de la ciudad de Granada. Se conformaron dos grupos experimentales: el grupo experimental 1, lo formaron 25 estudiantes y se trabajó con ellos la enseñanza del inglés mediante el uso de las TIC. El grupo experimental 2 lo conformaron 45 estudiantes y se usó una metodología tradicional en la enseñanza del inglés. La distribución del alumnado por condición y sexo puede observarse en la Tabla 1.

Tabla 1. Distribución de la muestra por condición y sexo

\begin{tabular}{lcccccc}
\hline & \multicolumn{2}{c}{ Niños } & \multicolumn{2}{c}{ Niñas } & \multicolumn{2}{c}{ Total } \\
\cline { 2 - 7 } \multicolumn{1}{c}{ Condición } & $n$ & $\%$ & $n$ & $\%$ & $n$ & $\%$ \\
\hline Grupo experimental 1 & 12 & 17.14 & 13 & 18.58 & 25 & 35.72 \\
Grupo experimental 2 & 23 & 32.86 & 22 & 31.43 & 45 & 64.29 \\
Total & 35 & 50 & 35 & 50 & 70 & 100 \\
\hline
\end{tabular}




\section{Instrumentos}

En el presente estudio se utilizaron como variables dependientes las relacionadas con madurez neuropsicológica (lenguaje, visopercepción, función ejecutiva, memoria, ritmo y lateralidad), atención, motivación y aprendizaje del inglés. El momento de evaluación (pretest-postest), el sexo (niño-niña) y la condición de los participantes (experimental 1- experimental 2) fueron utilizados como variables independientes o de agrupación.

\section{Aprendizaje del inglés}

Para evaluar el aprendizaje del inglés se utilizaron dos pruebas escritas "The Misterious Egypt", una para cada momento de evaluación (pre-post) construida ad hoc por el tutor, con un formato similar a las pruebas realizadas por el alumnado durante el curso académico para evaluar los aprendizajes en la materia.

La prueba consta de tres grandes escalas:

- Listening (compuesta por tres actividades): mide comprensión auditiva del vocabulario y gramática, estudiados en dicha unidad mediante a) la identificación de imágenes tras escuchar frases en inglés relacionadas con dichas imágenes, b) la identificación de imágenes después de escuchar una historia en inglés y c) la elección de la respuesta correcta en una actividad de opción múltiple tras escuchar una nueva historia en inglés.

- Reading (formada por dos pruebas): mide la comprensión lectora de un texto dado en inglés, mediante a) un cuestionario de elección múltiple ( 3 opciones de respuesta) y b) una batería de 8 preguntas de respuesta libre.

- Writing (consta de 4 pruebas): mide el vocabulario y los aspectos gramaticales trabajados a lo largo de la unidad a través de la identificación de imágenes y la cumplimentación de frases y textos en inglés.

\section{Madurez neuropsicológica}

La madurez neuropsicológica fue medida mediante el Cuestionario de Madurez Neuropsicológica escolar: CUMANES de Portellano et al. (2012). Este cuestionario permite elaborar una evaluación global del desarrollo neuropsicológico en alumnos y alumnas de primaria. Según los autores, la prueba presenta un alfa de Cronbach de .79 y está compuesto por una batería de 12 pruebas agrupadas en 6 secciones: 
Sección 1: Lenguaje. Consta de 6 pruebas:

- Comprensión audioverbal ( $\alpha=.67)$ : permite evaluar el lenguaje comprensivo, la atención sostenida y la memoria verbal.

- Comprensión de imágenes $(\alpha=.80)$ : evalúa el lenguaje comprensivo y la atención sostenida.

- Fluidez fonológica ( $\alpha=.64)$ : evalúa el lenguaje expresivo, la fluidez verbal, la función ejecutiva y la memoria de trabajo.

- Fluidez semántica ( $\alpha=.64)$ : permite evaluar el lenguaje expresivo, la fluidez verbal, la función ejecutiva y la memoria de trabajo.

- Leximetría ( $\alpha=.61$ ): evalúa la codificación lectora, la fluidez lectora, la atención sostenida y la memoria verbal.

- Escritura audiognósica ( $\alpha=.81$ ): evalúa la memoria a corto plazo, la atención sostenida, la eficiencia psicomotora, la visopercepción y el control grafomotor.

Sección 2: Visopercepción. ( $\alpha=.85)$ : permite evaluar la estructuración espacial, praxias constructivas, orientación espacial y el control grafomotor.

Sección 3: Función ejecutiva: evalúa la capacidad para la programación, la atención sostenida, la atención dividida, la memoria de trabajo, la memoria prospectiva, la capacidad de aprendizaje perceptivo motor, la flexibilidad mental y la capacidad de inhibición.

Sección 4: Memoria. Consta de dos pruebas:

- Memoria verbal $(\alpha=.68)$ : evalúa memoria explícita, memoria a corto plazo y memoria semántica.

- Memoria visual $(\alpha=.63)$ : evalúa memoria explícita, memoria a corto plazo, memoria no verbal y organización espacial.

Sección 5. Ritmo ( $\alpha=.83)$ : evalúa secuenciación, memoria sensorial, memoria a corto plazo, memoria implícita y atención sostenida.

Sección 6. Lateralidad ( $\alpha=.85)$ : evalúa dominancia del lenguaje, maduración neurológica, esquema corporal, organización espacial y destreza motriz. 
Los índices de fiabilidad de la prueba, para los participantes del estudio fueron similares a los que presentaba la prueba original.

\section{Atención}

La atención fue evaluada a través del cuestionario "CARAS-R. Test de percepción de diferencias" de Thurstone y Yela (2012). El cuestionario permite evaluar las aptitudes perceptivas y atencionales a través de 60 elementos gráficos compuestos por dibujos de caras con trazos básicos. El alumnado debe identificar la cara diferente de entre tres caras dadas y tacharla. Su aplicación puede ser individual o colectiva, aunque para este estudio se realizó de forma colectiva, en un tiempo de 3 minutos. El cuestionario nos permite obtener cuatro puntuaciones:

1. Aciertos (A). Número total de aciertos del sujeto. Se concederá un punto por cada una de las caras que aparecen tachadas en la hoja de copia (respuestas correctas). El número total de marcas correctas será el número de aciertos.

2. Errores (E). Número total de errores del sujeto. Se contará cada una de las cruces que haya marcado el sujeto y que no corresponda con la cara diferente de entre las tres. El número total de marcas erróneas será el número de errores.

3. Aciertos netos (A-E). Se calcula restando al número total de aciertos el número total de errores del sujeto. De esta forma se obtiene una medida más precisa de la eficacia en sus respuestas a la prueba.

4. Índice de Control de la Impulsividad (ICI). Se calcula dividiendo el número de Aciertos netos $(\mathrm{A}-\mathrm{E})$ entre el número de respuestas dadas por el sujeto $(\mathrm{A}+\mathrm{E})$.

\section{Motivación}

La motivación fue evaluada a través de una pregunta construida ad hoc, respuesta Likert de diez puntos donde $1=$ nada y $10=$ mucho. La pregunta mide la motivación del alumnado sobre la metodología llevada a cabo para la enseñanza del inglés, en el tiempo del estudio. Concretamente, la pregunta realizada a los estudiantes fue la siguiente: “¿Cuánto te ha gustado la manera de enseñar este tema de inglés y los recursos usados en él?”. 


\section{Procedimiento}

Para la realización del presente estudio, en primer lugar, se solicitó permiso al centro implicado. Una vez obtenidos los permisos correspondientes, tanto del profesorado como de los padres y de las madres de los participantes, se les explicaron los objetivos de la investigación al profesorado del $5^{\circ}$ curso de Educación de Primaria. Una vez conseguido el consentimiento informado, se procedió a la explicación y cumplimentación de los cuestionarios por parte del alumnado. Los cuestionarios fueron cumplimentados por el alumnado en diferentes sesiones.

El cuestionario usado para evaluar el aprendizaje del inglés fue administrado por el especialista de inglés del centro en dos fases, pretest y postest. Las pruebas utilizadas en ambas fases difieren en el orden de los ítems de cada una de las actividades, así como los textos usados para la comprensión oral y escrita, con la finalidad de no generar aprendizaje por recuerdo. El intervalo entre ambas fases fue de tres semanas durante las cuales se produjo la intervención en ambos grupos. Como se ha comentado con anterioridad, el grupo 1 difiere del grupo 2 en la metodología usada para la enseñanza del inglés. Concretamente, en el grupo 1 se utilizó las TIC (uso de ordenador, juegos interactivos, DVD, CD ROM, videojuegos y páginas webs) para la enseñanza del inglés y en el grupo 2 se utilizó una metodología tradicional (basada en el uso del libro de texto y las fichas).

Entre las actividades utilizadas en el grupo 1 destaca la utilización de un videojuego creado para la presente investigación. Se trata de un juego online donde el docente crea grupos de trabajo con sus alumnos y alumnas y asigna a cada uno un vocabulario específico o grupal. En el juego, el alumnado se va encontrando a rivales a los que debe desarmar traduciendo palabras del español al inglés y viceversa o utilizando de manera correcta frases gramaticales. Las palabras de vocabulario y las frases gramaticales son creadas por el docente, lo que facilita que se vayan modificando cuando el alumnado las vaya adquiriendo. Cuando el alumnado utiliza de manera correcta la gramática o las palabras, va obteniendo puntos y pasando de pantalla. Con esos puntos, el alumnado tiene la opción de ir a una cripta y cambiarlos por accesorios. Además, la utilización correcta del inglés permite al alumnado subir de nivel e ir mejorando su personaje. Igualmente, el alumnado puede seguir jugando en casa y elegir al rival concreto al que quiere enfrentarse. 
Otra actividad destacada, fue la realización de videoconferencias con alumnos y alumnas de un colegio de Manchester, en las que se proponía diferentes temas (como por ejemplo salud, cultura, reciclaje...) y el alumando tenía que realizar preguntas y respuestas en inglés sobre la temática propuesta.

Por otra parte, la cumplimentación del Cuestionario de Madurez Neuropsicológica escolar se realizó en la fase pretest de manera individual siguiendo el orden establecido por los autores de dicho manual para cada una de las pruebas. La prueba fue administrada por el orientador del centro en distintas sesiones, en las primeras horas del horario escolar, para evitar el efecto del cansancio en el alumnado participante.

El Test de percepción de diferencias fue administrado en la fase pretest y la cuestión sobre la motivación en la fase postest. Ambos cuestionarios fueron administrados de manera colectiva por evaluadores entrenados para tal fin.

Una vez cumplimentados los cuestionarios por el alumnado se procedió al análisis de los resultados y a la redacción del informe. Por último, se informó al centro sobre los resultados obtenidos.

\section{Análisis de datos}

Teniendo como premisa los objetivos e hipótesis de la presente investigación, la metodología utilizada fue de tipo cuasi-experimental, con dos grupos experimentales (grupo experimental 1-grupo experimental 2) y dos momentos temporales (pretest y postest). No fue posible la asignación aleatoria a las dos condiciones experimentales, si no que se utilizaron grupos-clase ya formados.

En relación con los análisis preliminares, además de obtener las medidas de tendencia central y dispersión, para comprobar las diferencias o semejanzas entre los grupos experimental 1 y experimental 2 en la fase pre de la evaluación, se realizó la prueba $t$, para muestras independientes, más adecuada en función de la homogeneidad de las varianzas.

Posteriormente, se realizó una ANOVA mixto de medidas repetidas en el que se incluyó como factor intra-grupo el momento de la evaluación (pretest-postest) y como factor entregrupos la condición (experimental 1-experimental 2). Seguidamente, se realizó la prueba $t$ de 
student para muestras independientes para comprobar en qué momento de la evaluación se encuentran las posibles diferencias entre los grupos.

Igualmente, se realizaron correlaciones de Pearson para analizar la relación entre el aprendizaje del inglés y las variables intrapersonales analizadas. Esto es, la madurez neuropsicológica, la atención y la motivación.

Los diferentes análisis se realizaron con el programa estadístico Statistical Pack ageforthe Social Sciences (SPSS) en su versión Stadistic 20.0 para Windows.

\section{Resultados}

\section{Aprendizaje del inglés y metodología}

Debido a que los participantes en la investigación no habían sido asignados aleatoriamente a las dos condiciones experimentales, se realizó una prueba de diferencias de medias, para comprobar si ambos grupos partían de niveles similares de inglés en la fase pre de la evaluación. Los resultados muestran que, antes de la fase de intervención, ambos grupos obtienen puntuaciones similares en las pruebas listening $\left(t_{(68)}=1.03, p=.308\right)$, reading $\left(t_{(68)}=1.69\right.$, $p=.096)$, writing $\left(t_{(68)}=1.40, p=.167\right)$ y en el el total de la prueba de inglés $\left(t_{(68)}=.19, p=.849\right)$.

Por otra parte, los resultados obtenidos en la ANOVA de medidas repetidas en las variables de aprendizaje del inglés muestran efectos principales del factor momento de la evaluación (pre-post) en todas las variables de aprendizaje del inglés analizadas: listening $F_{(1.66)}=231.14, p=.000$; reading $F_{(1.66)}=41.84, p=.000$; writing $F_{(1.66)}=310.80, p=.000$; y en el total de la prueba de inglés $F_{(1.66)}=371.49, p=.000$. El alumnado de ambos grupos (experimental y control) obtiene puntuaciones significativamente más elevadas en la fase postintervención (ver Tabla 2). Se observan unos tamaños del efecto elevados para el total de aprendizaje del inglés (eta $\left.{ }^{2}=.85\right)$ y para las pruebas listening $\left(\right.$ eta $\left.^{2}=.78\right)$ y writing $\left(\right.$ eta $\left.^{2}=.83\right)$, y un tamaño medio del efecto para la prueba reading $\left(\mathrm{eta}^{2}=.39\right)$. 
Tabla 2. Medias y desviaciones típicas de las variables del aprendizaje del inglés en la fase pre y post evaluación

\begin{tabular}{|c|c|c|c|c|c|c|c|c|c|c|c|c|}
\hline & \multicolumn{4}{|c|}{ Experimental 1} & \multicolumn{4}{|c|}{ Experimental 2} & \multicolumn{4}{|c|}{ Total } \\
\hline & \multicolumn{2}{|c|}{ Pre } & \multicolumn{2}{|c|}{ Post } & \multicolumn{2}{|c|}{ Pre } & \multicolumn{2}{|c|}{ Post } & \multicolumn{2}{|c|}{ Pre } & \multicolumn{2}{|c|}{ Post } \\
\hline & $M$ & $S d$ & $M$ & $S d$ & $M$ & $S d$ & $M$ & $S d$ & $M$ & $S d$ & $M$ & $S d$ \\
\hline $\begin{array}{l}\text { Liste- } \\
\text { ning }\end{array}$ & .38 & .23 & .79 & .16 & .43 & .22 & .66 & .17 & .41 & .22 & .71 & .18 \\
\hline Reading & .47 & .17 & .65 & .22 & .39 & .18 & .50 & .22 & .42 & .18 & .55 & .23 \\
\hline Writing & .13 & .12 & .63 & .22 & .17 & .11 & .40 & .21 & .15 & .12 & .48 & .24 \\
\hline Total & .32 & .13 & .68 & .17 & .33 & .15 & .52 & .18 & .33 & .14 & .58 & .19 \\
\hline
\end{tabular}

Los resultados muestran efectos de interacción significativos entre los factores momento de evaluación (pre-post) y condición (experimental 1- experimental 2) en las variables de aprendizaje del inglés: listening $F_{(1.66)}=18.14$, $p=.000$, writing $F_{(1.66)}=43.62$, $p=.000$, y total de la prueba de inglés $F_{(1.66)}=37.39, p=.000$. Los tamaños del efecto de la interacción son moderados para el total de la prueba de inglés $\left(\mathrm{eta}^{2}=.36\right)$, y para las pruebas listening $\left(\mathrm{eta}^{2}=.22\right)$ y writing $\left(\right.$ eta $\left.^{2}=.40\right)$. Sin embargo, el tamaño del efecto es bajo en la prueba reading $\left(\right.$ eta $^{2}=$ $.04)$.

En línea con los efectos de interacción, la prueba de diferencia de medias, para muestras independientes, revela que existen diferencias significativas entre los grupos experimental 1 y el experimental 2 en la fase post-evaluación. Los niños y niñas del grupo experimental 1 obtienen puntuaciones más elevadas que sus iguales del grupo experimental 2 en el listening $t_{(69)}=2.84, p=.006$, writing $t_{(69)}=4.22, p=.000$ y en el total de la prueba de inglés $t_{(69)}=3.67$, $p=.000$. Por otra parte, no se encontraron efectos de interacción en la prueba reading $F_{(1.66)}=2.58, p=.113$, aunque las prueba de diferencia de medias revela que existen diferencias significativas entre los grupos experimental 1 y el experimental 2 en la fase post-evaluación. El alumnado del grupo experimental 1 obtienen puntuaciones más elevadas que sus iguales del grupo experimental 2 en la prueba reading $t_{(69)}=2.65, p=.010$.

\section{Aprendizaje del inglés y madurez neuropsicológica}

Con el fin de analizar la relación existente entre la madurez neuropsicológica y el aprendizaje del inglés se realizó un análisis de correlación de Pearson entre estas dos variables (ver Tabla 3). Los datos obtenidos señalan la presencia de una correlación positiva y significativa entre el listening y la comprensión audioverbal. De igual forma, el listening correlacionó de manera positiva y significativa con la comprensión de imágenes, con leximetría (palabras por minuto), con la escritura audiognósica y con el total de madurez neuropsicológica. 
Así mismo, la variable listening correlacionó negativamente y de manera significativa con la función ejecutiva (tiempo en segundos) y con la función ejecutiva (número de errores). No se encontraron correlaciones significativas entre el listening y el resto de variables del Cumanes, como son la fluidez fonológica y semántica, la leximetría (respuestas correctas), la visopercepción, las memorias verbal y visual, el ritmo o las lateralidades manual, ocular o podálica.

Tabla 3. Correlaciones de Pearson entre aprendizaje del inglés y madurez neuropsicológi$c a$

\begin{tabular}{lcccc}
\hline \multirow{2}{*}{ Madurez neuropsicológica } & \multicolumn{4}{c}{ Aprendizaje del inglés } \\
\cline { 2 - 5 } & Listening & Reading & Writing & Total \\
\hline Comprensión audioverbal & $.31^{*}$ & $.27^{*}$ & .14 & $.30^{*}$ \\
Comprensión de imágenes & $.53^{* *}$ & $.24^{*}$ & $.29^{*}$ & $.44^{* *}$ \\
Fluidez fonológica & -.01 & -.09 & .08 & -.02 \\
Fluidez semántica & .16 & -.01 & .13 & .11 \\
Leximetría (Respuestas correctas) & -.01 & .15 & -.05 & .04 \\
Leximetría (Palabras por minuto) & $.40^{* *}$ & $.36^{* *}$ & $.35^{* *}$ & $.45^{* *}$ \\
Escritura audiognósica & $.38^{* *}$ & $.45^{* *}$ & $.36^{* *}$ & $.47^{* *}$ \\
Visopercepción & .11 & .19 & .10 & .16 \\
Función ejecutiva (Tiempo en segundos) & $-.31^{* *}$ & $-.37^{* *}$ & $-.38^{* *}$ & $-.41^{* *}$ \\
Función ejecutiva (Número de errores) & $-.26^{* *}$ & $-.26^{*}$ & -.23 & $-.30^{*}$ \\
Memoria verbal & .14 & .03 & .09 & .11 \\
Memoria visual & .16 & -.02 & .04 & .08 \\
Ritmo & .21 & .12 & .03 & .16 \\
Lateralidad Manual & .05 & -.02 & -.02 & .01 \\
Lateralidad Ocular & -.01 & -.03 & -.01 & -.02 \\
Lateralidad podálica & .05 & .07 & .06 & .07 \\
Total madurez neuropsicológica & $.52^{* *}$ & $.34 * *$ & $.33^{* *}$ & $.49^{* *}$ \\
\hline
\end{tabular}

$* \mathrm{p}<.05$ (bilateral); $* * \mathrm{p}<.01$ (bilateral)

Por otra parte, se observó que la prueba reading correlacionó positivamente y de manera significativa con la comprensión audioverbal, con la comprensión de imágenes, con leximetría (palabras por minuto), con la escritura audiognósica y con el total de madurez neuropsicológica. Del mismo modo, se observan correlaciones negativas y significativas con la función ejecutiva (tiempo en segundos) y la función ejecutiva (número de errores). No se encontraron correlaciones significativas entre el reading y las variables de madurez neuropsicológica relacionadas con la fluidez fonológica y semántica, la leximetría (respuestas correctas), la visopercepción, las memorias verbal y visual, el ritmo o las lateralidades manual, ocular o podálica. 
Por otro lado, los datos indican que el writing correlacionó positivamente y de manera significativa con la comprensión de imágenes, con leximetría (palabras por minuto), con la escritura audiognósica y con el total de madurez neuropsicológica. Del mismo modo, existen correlaciones negativas y significativas con la función ejecutiva (tiempo en segundos). No se encontraron correlaciones significativas entre el writing y las variables relacionadas con comprensión audioverbal, la fluidez fonológica y semántica, la leximetría (respuestas correctas), la visopercepción, la función ejecutiva medida con el número de errores, las memorias verbal y visual, el ritmo o las lateralidades manual, ocular o podálica.

La puntuación total obtenida en el test de inglés correlacionó positivamente y de manera significativa con la comprensión audioverbal, con la comprensión de imágenes, con leximetría (palabras por minuto), con la escritura audiognósica y con el total de madurez neuropsicológica. Del mismo modo, existen correlaciones negativas y significativas entre la puntuación total del test de inglés con la función ejecutiva (tiempo en segundos) y la función ejecutiva (número de errores).No se encontraron correlaciones estadísticamente significativas con las variables de madurez neuropsicológica relacionadas con la fluidez fonológica y semántica, la leximetría (respuestas correctas), la visopercepción, las memorias verbal y visual, el ritmo o las lateralidades manual, ocular o podálica.

\section{Aprendizaje del inglés y atención}

La Tabla 4 recoge los resultados obtenidos de la realización de la correlación de Pearson entre el aprendizaje del inglés y la atención. De esta manera, se observan correlaciones significativas positivas entre todas las variables del aprendizaje del inglés analizadas (listening, reading, writing y total obtenido en la prueba de inglés) y el número de aciertos. Igualmente, se produce una correlación positiva y significativa entre las variables de aprendizaje del inglés y el número de aciertos netos. Por el contrario, no se encontraron correlaciones significativas entre las variables del aprendizaje del inglés y los errores ni con el índice de control de impulsividad. 
Tabla 4. Correlaciones entre aprendizaje del inglés y atención

\begin{tabular}{lcccc}
\hline \multirow{2}{*}{ Atención } & \multicolumn{4}{c}{ Aprendizaje del inglés } \\
\cline { 2 - 5 } & Listening & Reading & Writing & Total \\
\hline Aciertos & $.38^{* *}$ & $.37^{* *}$ & $.43^{* *}$ & $.46^{* *}$ \\
Errores & -.01 & -.08 & .01 & -.04 \\
$\begin{array}{l}\text { Aciertos netos } \\
\text { Índice de control de impul- } \\
\text { sividad }\end{array}$ & $.37^{* *}$ & $.37^{* *}$ & $.41^{* *}$ & $.45^{* *}$ \\
\hline$* \mathrm{p}<.05$ (bilateral) $* * * \mathrm{p}<.01$ (bilateral) & .03 & .14 & .06 & .09 \\
\hline
\end{tabular}

$* \mathrm{p}<.05$ (bilateral); $* * \mathrm{p}<.01$ (bilateral)

\section{Aprendizaje del inglés y motivación}

Las medias y desviaciones típicas en motivación se muestran en la Tabla 5. Los resultados obtenidos de la prueba de diferencia de medias en motivación entre la condición (experimental 1 y experimental 2) en la fase post-intervención muestra diferencias significativas entre ambos grupos, obteniendo una puntuación mayor en motivación el grupo experimental 1 con respecto al grupo experimental $2 t_{(51.18)}=19.18, p=.000$. Obtenido el tamaño del efecto de la diferencia, a través de eta cuadrado y el coeficiente de correlación, los resultados muestran un tamaño del efecto muy elevado eta $^{2}=.75$ y r $=.90$.

Tabla 5. Medias y desviaciones típicas en motivación

\begin{tabular}{ccccccc}
\cline { 2 - 6 } & \multicolumn{2}{c}{ Experimental 1 } & \multicolumn{2}{c}{ Experimental 2 } & \multicolumn{2}{c}{ Total } \\
\cline { 2 - 7 } & $M$ & $S d$ & $M$ & $S d$ & $M$ & $S d$ \\
\hline Motivación & 9.80 & 0.41 & 3.74 & 2.07 & 5.87 & 3.36 \\
\hline
\end{tabular}

\section{Discusión y Conclusiones}

En primer lugar, los resultados de este estudio muestran que, independientemente de que el método utilizado por el profesorado sea una enseñanza tradicional o una docencia asistida por las nuevas tecnologías, el alumnado mejora su aprendizaje después de la acción educativa. El alumnado de ambos grupos experimentales obtiene puntuaciones significativamente superiores después de las sesiones de aprendizaje de la materia. No obstante, y a pesar de que existen estudios que cuestionan la eficacia de las TIC en el aprendizaje (Condie y Munro 2007; Cuban y Kirkpatrick, 1998), los análisis preliminares confirman la primera hipótesis de partida del presente estudio, la enseñanza del inglés a través de las TIC mejora el aprendizaje del alumnado frente a la enseñanza tradicional. Estos resultados también fueron encontrados en el área de las matemáticas por otro estudio llevado a cabo por Cheung y Slavin (2013), 
quienes hallaron que las TIC producen efectos positivos en comparación con la enseñanza tradicional en el aprendizaje de las matemáticas. En esta misma línea, Cox et al. (2003), encontraron la presencia de un efecto positivo de las TIC en el aprendizaje del alumnado de las materias básicas. No obstante, señalaron que este efecto depende de los recursos tecnológicos empleados y de la materia en cuestión.

Estos resultados son congruentes si se tiene en consideración que la irrupción de las tecnologías en el ámbito educativo, han generado un incremento en la motivación y en el interés del alumnado en el proceso de aprendizaje y han favorecido el desarrollo de una mayor autonomía y competencia ante una sociedad en continuo cambio y transformación. La cantidad de tiempo que el alumnado dedica al uso de determinadas tecnologías (teléfono móvil, internet, redes sociales y videojuegos) fuera del contexto escolar y la variedad de recursos digitales y audiovisuales que se están desarrollando, lleva a que los docentes se planteen la necesidad de introducir estas tecnologías en sus metodologías de enseñanza. Por ello, elaborar y hacer uso de los recursos tecnológicos (tales como los videojuegos) que potencian el aprendizaje del alumnado, tanto dentro como fuera del aula, tendría que ser un aspecto vital para tener en cuenta por los docentes. Además, esta metodología de enseñanza a través de TIC, favorece la autonomía del alumnado, la colaboración entre ellos, y la creación de un discurso dentro y fuera del aula que potencia el aprendizaje y a su vez, favorece que este aprendizaje se realice de forma más lúdica e independiente. En esta línea, Ghasemi y Hashemi (2011) señalan que el aprendizaje del inglés como segunda lengua, a través de la utilización de las TIC, no solo mejora la motivación de los estudiantes, sino que potencia el aprendizaje interactivo, la adaptación a los diferentes estilos de aprendizaje del alumnado y proporciona mayores oportunidades para el desarrollo de la creatividad. De esta forma, se proporcionará la oportunidad al alumnado de que, a través de la utilización de videojuegos, chat u otros recursos, sean partícipes activos de su propio aprendizaje del inglés.

Por otra parte, los resultados del estudio confirman la segunda hipótesis ya que existe una relación positiva entre la madurez neuropsicológica del alumnado y su aprendizaje del inglés. El alumnado que obtiene puntuaciones altas en las pruebas listening, reading y writing también obtienen puntuaciones elevadas en madurez neuropsicológica y en algunas de las variables que la conforman. Más concretamente, los resultados muestran que hay determinadas variables de madurez neuropsicológicas que tienen una mayor influencia en el aprendizaje del inglés (Keane, Keane, y Blicblau, 2016). En este sentido, parece que la comprensión tanto 
audiovisual como de imágenes, la velocidad lectora, la capacidad para copiar palabras y frases, así como la función ejecutiva, son las variables que mayor influencia tienen en el aprendizaje del inglés como segunda lengua. Sin embargo, otras variables como la fluidez fonológica o semántica, la visopercepción, la memoria, el ritmo o la lateralidad no parecen tener relación con el aprendizaje del inglés.

De forma similar, como ocurriría en cualquier contenido de aprendizaje, la atención muestra tener una relación con el aprendizaje del inglés, confirmando la tercera hipótesis del estudio. Los niños y niñas que tienen puntuaciones altas en las pruebas de inglés realizadas, también obtienen puntuaciones altas en número de aciertos y bajas en errores en el cuestionario de atención. Tal y como señala Ortiz (2009) uno de los papeles fundamentales de la atención en el campo de la educación es su influencia en el control de los comportamientos, aprendizajes, procesos cognitivos y, en general, en las actividades relacionadas con la vida escolar. En esta línea, Vassilopoulou y Mavrikaki (2016) realizaron un estudio en el que mostraron que el uso de las TIC logró atraer la atención del alumnado y mantenerlos más activos en el proceso de aprendizaje que la utilización de una metodología tradicional. Por esta razón, parece fundamental, fomentar una metodología activa apoyada en las TIC, puesto que este tipo de metodología facilita los procesos atencionales y, por tanto, se favorece el aprendizaje del alumnado.

Por otra parte, los resultados confirman la cuarta hipótesis, ya que muestran que el alumnado que aprende inglés a través de TIC, presenta una mayor motivación hacia la materia que se le ha enseñado, que el alumnado que recibe una enseñanza tradicional. Resultados similares se han encontrado en otros estudios como el realizado por Tüzün, Y1lmaz-Soylu, Karakuş, İnal, y Kızılkaya, (2009), aunque en este caso la materia a enseñar fuese geografía. Los autores mostraron que al comparar la motivación de los estudiantes en geografía, utilizando una metodología tradicional frente a otra basada en el uso del videojuego, como recurso tecnológico, aquellos estudiantes que aprendieron a través del uso de videojuegos obtuvieron una motivación significativamente superior, así como una mejor calificación que aquellos que recibieron una enseñanza tradicional.

Igualmente, una metodología basada en las TIC en la enseñanza de segundas lenguas favorece el interés hacia el aprendizaje, facilita el acceso a otras culturas, mejora la interacción entre el alumnado, la independencia en su propio aprendizaje, así como su motivación 
hacia el mismo. Todos estos efectos positivos sugieren que la incorporación de las TIC como herramienta en los entornos de aprendizaje tiene una gran utilidad para apoyar y favorecerla adquisición de segundas lenguas (Adedokun-Shittu y Shittu, 2015).

De esta manera, se pueden extraer las siguientes conclusiones de la presente investigación: a) la metodología empleada influye en el aprendizaje del inglés como segunda lengua. Concretamente, la utilización de las TIC favorece la adquisición del inglés frente a la enseñanza tradicional; b) existe una relación entre la madurez neuropsicológica y el aprendizaje del inglés, teniendo especial relevancia la función ejecutiva, la comprensión audiovisual y de imágenes, la velocidad lectora y la capacidad para copiar frases y palabras; c) Al igual que ocurre en otras áreas de conocimiento, la atención es fundamental para la adquisición del inglés y d) la motivación del alumnado se ve incrementada cuando se utiliza una enseñanza basada en las TIC frente a una enseñanza tradicional.

No obstante, se deben tener en cuenta las limitaciones del estudio, muchas de ellas están relacionadas con la naturaleza del diseño y la forma de ejecución del estudio. No fue posible asignar al azar al alumnado el grupo experimental 1 y en el grupo experimental 2 y por lo tanto no se pueden establecer relaciones de causalidad, ni se controlaron otro tipo de variables individuales tales como el grupo cultural o étnico de los participantes o el rendimiento previo. No obstante, se ha intentado controlar otro tipo de limitaciones asociadas a los diseños cuasiexperimentales a través de la utilización de dos grupos experimentales distintos y de diferentes momentos temporales en la evaluación de los sujetos. Asimismo, se realizó una prueba $t$ para comprobar si existían diferencias entre los grupos experimental 1 y experimental 2 antes de la intervención, con el fin de considerar las diferencias iniciales y realizar los análisis pertinentes.

Por otro lado, no se puede perder de vista que el estudio se ha realizado con un número de sujetos muy reducido y en un centro específico de la ciudad de Granada. Por esta razón, la validez externa es escasa y es muy complejo extraer conclusiones firmes y generalizar los resultados a otros contextos. Igualmente, la motivación ha sido evaluada a través de una única pregunta para conocer la satisfacción. 
Del mismo modo, otra limitación viene dada por la utilización de un estudio de corte transversal. Concretamente, se miden los efectos de la intervención a corto plazo, impidiendo comprobar el impacto real del uso de las TIC en la enseñanza.

Como prospectiva, en futuras investigaciones sería interesante comparar si la utilización de diferentes recursos TIC, como por ejemplo videojuegos vs pizarra digital influyen de manera diferenciar en el aprendizaje del inglés y en la motivación del alumnado. Igualmente, sería interesante realizar un análisis longitudinal del efecto del uso de las TIC en todas las etapas educativas y en diferentes materias. Asimismo, se podría analizar si las TIC fomentan el manteniento de la atención y de esta manera, favorencen el aprendizaje.

\section{Referencias}

Abdel-Rahman, A. (2009). Attitudes of Jordanian College Students towards Learning English as a Foreign Language. College Student Journal, 43(2), 682-694.

Adedokun-Shittu, N.A. y Shittu, A.J.K. (2015). Assessing the impacts of ICT deployment in teaching and learning in higher education: Using ICT impact assessment model. Journal of Applied Research in Higher Education, 7(2), 180-193. doi:10.1108/JARHE-022013-0012

Arteaga, C. (2011). Uso de las TIC Para El Aprendizaje del inglés en la Universidad Autónoma de Aguascalientes. Apertura, 3(2), 1-13.

Badia, A., Meneses, J. y Sigalés, C. (2013). Percepción de los docentes sobre los factores que afectan el uso educativo de las TIC en el aula equipada de tecnología. Electronic Journal of Research in Educational Psychology,11(31), 787-808. doi:10.14204/ejrep.31.13053

Boujon, C.H. y Quaireau, C.H. (1999). Atención, aprendizaje y rendimiento escolar. Madrid: Narcea.

Cheung, A.C. y Slavin, R.E. (2013). The effectiveness of educational technology applications for enhancing mathematics achievement in K-12 classrooms: a meta-analysis. Educational Research Review, 9, 88-113. doi:10.1016/j.edurev.2013.01.001 
Condie, R. y Munro, B. (2007). The impact of ICT in schools - A landscape review. London: Becta.

Cox, M., Abbott, C., Webb, M., Blakeley, B., Beauchamp, T. y Rhodes, V. (2003). ICT and attainment: A review of the research literature. ICT in Schools Research and Evaluation Series. London: Becta.

Cuban, L. y Kirkpatrick, H. (1998). Computers make kids smarter-right? Technos, 7(2), 2631.

Diamond, A. (2013). Executive Functions. Annual Review of Psychology, 64, 135-168. doi:10.1146/annurev-psych-113011-143750

Domalewska, D. (2014). Technology-supported classroom for collaborative learning: Blogging in the foreign language classroom. International Journal of Education and Development using Information and Communication Technology, 10(4), 21-30.

Emir, S.E. (2010). A Study on the Affective Attitudes of Students at Gazi Educational Faculty towards English Classes. Ekev Academic Review, 14(45), 129-136.

Faradilla, E. (2010). Attitude towards Use of ICT and Its Actual Level of Use in English Language Teaching in Miri, Sarawak. Asian Social Science, 8(11), 8-12.

Friedrich, P. y Matsuda, A. (2010). When five words are not enough: a conceptual and terminological discussion of English as a lingua franca. International Multilingual Research Journal, 4, 20-30.

García-Sevilla, J. (1997). Psicología de la atención. Madrid: Síntesis.

Ghasemi, B. y Hashemi, M. (2011). ICT: New wave in english language learning/teaching. Procedia - Social and Behavioral Sciences, 15(0), 3098-3102. doi:10.1016/j.sbspro.2011.04.252

González-Castro, P., Álvarez, L., Núñez, J.C., González-Pienda, J.A. y Álvarez, D. (2008). La aplicación de una estrategia computarizada para enseñar y aprender matemáticas en Educación Primaria. European Journal of Education and Psychology, 1(1), 21-38.

Granito, M., y Chernobilsky, E. (2012). The effect of technology on a student's motivation and knowledge retention. Proceedings of Northeastern Educational Research Association Conference (NERA, 2012) (pp. 1-22). Paper 17. Recuperado de http://digitalcommons.uconn.edu/nera_2012/17 
Harrison, C., Comber, C., Fisher, T., Hawe, K., Lewin, C., Lunzer, E., ... Watling, R. (2002). ImpaCT2: The Impact of Information and Communication Technologies on Pupils Learning and Attainment. ICT in Schools Research and Evaluation Series. Londres: Becta.

Harutyunyan, P., Moldoveanu, A., Moldoveanu, F. y Asavei, V. (2015). Health-related impact, advantages and disadvantages of ICT use in education, compared to their absence in the past. The International Scientific Conference eLearning and Software for Education, 1, 557-563.

Hernández, L.K. y Muñoz, L.F. (2012). Usos de las tecnologías de la información y la comunicación (TIC) en un proceso formal de enseñanza y aprendizaje en la Educación Básica. Zona Próxima, 16, 2-13.

Hollenbeck, J. y Hollenbeck, D. (2009). Using technology to bridge the cultures together in the multicultural classroom. In D. P. French, (Ed.). Technology in the College Science Classroom (pp. 1-6). Stillwater: Oklahoma State University.

Hubackova, S. y Ruzickova, M. (2011). Experience in foreign language teaching with ICT support. Procedia Computer Science, 3(0), 243-247. doi:10.1016/j.procs.2010.12.041

Keane, T., Keane, W.F. y Blicblau, A. (2016). Beyond traditional literacy: Learning and transformative practices using ICT. Education and Information Technologies, 21(4), 769-781. doi:10.1007/s10639-014-9353-5

Kolbakova, F. (2014). The Use of ICT among the Teachers of English in Estonia by Comparison with Europe and Asia (Master's thesis). University of Tartu, Tartu, Estonia. Recuperado http://dspace.ut.ee/bitstream/handle/10062/46990/Master's_Thesis_Kolbakova.pdf

Laberge, D. (1995). Attentional processing. Londres: Harvard University Press.

León, B. (2008). Atención plena y rendimiento académico en estudiantes de enseñanza secundaria. European Journal of Education and Psychology, 1(3), 17-26.

Martínez, I. (2015). Relación entre el desarrollo neuropsicológico y el rendimiento escolar en Primaria. (Tesis de maestría). Universidad Internacional de La Rioja, Logroño.

Matsuda, A. y Friedrich, P. (2011). English as an international language: A curriculum blueprint. World Englishes, 30(3), 332-344. doi:10.1111/j.1467-971X.2011.01717.x 
McKay, S. (2002). Teaching English as an International Language: Rethinking Goals and Approaches. Oxford: Oxford University Press.

Muslem, A., y Abbas, M. (2017). The effectiveness of immersive multimedia learning with peer support on English speaking and reading aloud. International Journal of Instruction, $10(1), 203-218$.

Nelson, R. (2011). Vigilance, expectancy, and noise: Attention in second language lexical learning and memory. Second Language Research, 27(2), 153-171. doi:10.1111/j.1467-971X.2011.01717.x

Nordin, N., Embi, M.A. y Yunus, M.M. (2010). Mobile learning framework for lifelong learning. Procedia -Social and Behavioral Sciences, 7, 130-138. doi:10.1016/j.sbspro.2010.10.019

Nordin, N., Hamzah, M.I., Yunus, M.M. y Embi, M.A. (2010). The mobile learning environment for the in-service school administrators. Procedia - Social and Behavioral Sciences, 7, 671-679. doi:10.1016/j.sbspro.2010.10.091

Ortiz, T. (2009). Neurociencia y educación. Madrid: Alianza Editorial.

Passey, D., Rogers, C., Machell, J. y McHugh, G. (2004). The Motivational effect of ICT on pupils. England: University of Lancaster.

Portellano, J.A., Mateos, R. y Martínez, R. (2012). Cuestionario de Madurez Neuropsicológica Escolar: CUMANES. Madrid: Tea ediciones.

Portellano, Mateos, Martínez, R., Tapia, A. y Granados, M. J. (2000). Cumanin. Cuestionario de Madurez Neuropsicológica Infantil. TEA Ediciones. Madrid.

Prato, A. y Mendoza, M. (2006). Opinión, conocimiento y uso de portales web para la enseñanza del inglés como lengua extranjera. Revista Venezolana de Información, tecnología y Conocimiento, 1, 49-61.

Rabah, J. (2015). Benefits and challenges of Information and Communication Technologies (ICT) integration in Québec English schools. TOJET: The Turkish Online Journal of Educational Technology, 14(2), 24-31.

Robinson, P. (2003). Attention and memory during SLA. En C, Doughty. y M, Long (Ed.), The handbook of second language acquisition (631-678). Malden, MA: Blackwell. doi:10.1002/9780470756492.ch19 
Røkenes, F.M. y Krumsvik, R.J. (2016). Prepared to teach ESL with ICT? A study of digital competence in Norwegian teacher education. Computers \& Education, 97, 1-20. doi:10.1016/j.compedu.2016.02.014

Salehi, H. y Salehi, Z. (2012). Integration of ICT in language teaching: Challenges and barriers. International Proceedings of Economics Development and Research, 27, 215-219.

Santa-Cruz, C. y Rosas, R. (2017). Mapping of Executive Functions. Estudios de Psicología, 38(2), 284-310. doi:10.1080/02109395.2017.1311459

Sekharan, G.K., Setia, R., Mohamad, R., Abdul, Z.B., Luqman, A., Vadeveloo, T. y Ngah, H. C. (2014). Attitude, parental encouragement and perception of the importance of english in english language learning. Asian Social Science, 10(3). doi:10.5539/ass.v10n3p1

Shamsudin, A. (2009). Teaching Effectiveness and Course Content Suitability- A Survey among KPLI English Language Trainees at The Kuala Terengganu. (Masters thesis). Universiti Teknologi, MARA.

Silviyanti, T. M. (2014). Looking into EFL students' perceptions in listening by using English movie videos on YouTube. Studies in English Language and Education, 1(1), 45-63.

Silviyanti, T. M., y Yusuf, Y. Q. (2015). EFL teachers' perceptions on using ICT in their teaching: To use or to reject?. Teaching English with Technology, 15(4), 29-43.

Thurstone, L.L. y Yela, M. (2012). CARAS-R. Test de percepción de diferencias. Madrid: Tea ediciones.

Tüzün, H., Yılmaz-Soylu, M., Karakuş, T., İnal, Y. y Kızılkaya, G. (2009). The effects of computer games on primary school students' achievement and motivation in geography learning. Computers \& Education, 52(1), 68-77. doi:10.1016/j.compedu.2008.06.008

Vassilopoulou, A., y Mavrikaki, E. (2016). Can ICT in biology courses improve AD/HD students' achievement?. Improving Schools, 19(3), 246-257.

Yunus, M.M., Chua, P.L., Maimun, A.L. y Rizauddin, R. (2010). Evaluation of ICT usage for general or English learning purposes. WSEAS Transactions on Information Science and Applications, 205-211. 
Yunus, M.M., Nordin, N., Salehi, H., Embi, M.A. y Salehi, Z. (2013). The use of information and communication technology (ICT) in teaching ESL writing skills. English Language Teaching, 6(7), 1-8. doi:10.5539/elt.v6n7p1

Yunus, M.M., Nordin, N., Salehi, H., Embi, M.A. y Salehi, Z. (2014). Future of ICT as a pedagogical tool in ESL teaching and learning. Research Journal of Applied Sciences, Engineering and Technology, 7(4), 764-770.

Yunus, M.M., Salehi, H. y Chenzi, C. (2012). Integrating social networking tools into ESL writing classroom: Strengths and weaknesses. English Language Teaching, 5(8), 4248. doi:10.5539/elt.v5n8p42

Recibido: $28-02-2018$

Aceptado: 08-12-2018 